\title{
26. Program Design using Structure Diagrams (Iteration)
}

If a main job can be broken down into a single simple task repeated a number of times - this is called an iteration (means repetition) and it can be shown on a Structure Diagram.

Example 1 - Display a screen of 24 'hello's (in a column).

Displaying a whole screen of 'hello's breaks down into the simpler task of displaying the message line 'Hello' - repeated 24 times. On a structure diagram this is shown as:

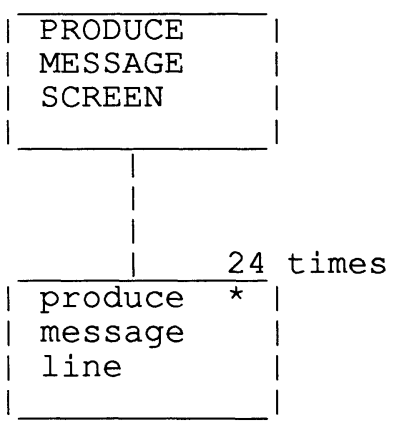

The asterisk in the top right corner of the lower rectangle indicates that the smaller task is repeated - and the number of times is shown above the rectangle.

Example 2 - Ask for a stream of numbers to be keyed in - stop when zero is typed.

This task will break down into the simpler task of programming the computer to ask for a single number - repeated until a zero is keyed.

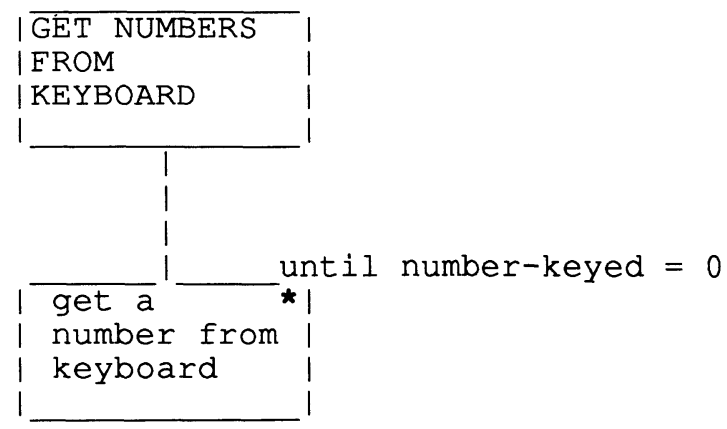

\section{Exercises}

Produce Structure Diagrams for each of the following tasks.

1. Clearing the screen consists of displaying 24 blank lines.

2. Homework consists of writing 100 lines saying 'I must work harder in class'. 